\title{
Do Conceito de Número e Magnitude na Matemática Grega Antiga*
}

\author{
Diego P. Fernandes**
}

\begin{abstract}
Resumo
O propósito deste texto é apresentar a evolução da relação entre o conceito de número e magnitude na matemática grega antiga. Revisaremos o programa pitagórico e a sua crise com a descoberta das magnitudes incomensuráveis. Em seguida, sumarizamos o trabalho de Eudoxus. Ele amplificou a teoria das proporções pitagórica, fazendo-a aplicável também a magnitudes incomensuráveis. Veremos que, com o passar do tempo, a existência de magnitudes incomensuráveis deixou de ser algo estranho. Já na época de Platão e Aristóteles, sua existência já era lugar comum: inclusive ao ponto de se considerar absurdo que todas as magnitudes fossem comensuráveis. Aristóteles criticou o programa pitagórico e defendeu que, apesar de pertencerem à mesma categoria (quantidade), número e magnitude são de espécie distinta: numero é discreto e magnitude é contínuo. Por fim, apresentamos brevemente como o conceito de numero foi amplificado com o passar dos séculos até incluir a noção de continuidade.
\end{abstract}

Palavras-chave: número, magnitude, incomensuráveis, matemática grega antiga.

\section{Abstract \\ The Concept of Number and Magnitude in Ancient Greek Mathematics}

The aim of this text is to present the evolution of the relation between the concept of number and magnitude in ancient Greek mathematics. We will briefly revise the Pythagorean program and its crisis with the discovery of incommensurable magnitudes. Next, we move to the work of Eudoxus and present its advances. He improved the Pythagorean theory of proportions, so

*Recibido: mayo 2017. Aceptado: junio 2017

**Universidad de Salamanca. Salamanca, España. Email: diegofernandess@gmail.com 
that it could also treat incommensurable magnitudes. We will see that, as the time passed by, the existence of incommensurable magnitudes was no longer something strange. Already in the period of Plato and Aristotle, their existence was common place: up to the point of being considered absurd that all magnitudes were commensurable. Aristotle criticized the Pythagorean program and defended that, though belonging to the same category (quantity), number and magnitude are of distinct species: number is discrete and magnitude is continuous. We finish by presenting briefly how the concept of number was amplified throughout the centuries until it came also to include the notion of continuity.

Keywords: number, magnitude, incommensurable, ancient Greek mathematics

\section{Contents}

1 Introdução

$\begin{array}{lll}2 & \mathrm{O} \text { conceito de número nos gregos } & 10\end{array}$

$\begin{array}{lll}3 & \text { O programa pitagórico } & 12\end{array}$

3.1 O número como essência das coisas . . . . . . . . . . . . . . . . . . 12

3.2 A descoberta da incomensurabilidade . . . . . . . . . . . . . . . . 13

4 Os incomensuráveis e a nova teoria das proporções 14

4.1 A teoria das proporções de Eudoxus . . . . . . . . . . . . . . 15

4.2 A existência de incomensuráveis como conhecimento básico . . . . . . 17

5 Aristóteles: quantidades contínuas e discretas 18

5.1 O contínuo não pode mesmo ser tornado discreto? . . . . . . . . . . . 19

Um contínuo é infinitamente divisível . . . . . . . . . . . 20

6 Considerações finais: de alguns desenvolvimentos posteriores do conceito de número

Referências 


\section{Introdução}

O conceito de número surgiu do processo de medição e permaneceu durante muito tempo associado a ele. Foi a partir das investigações dos membros da escola pitagórica que a associação do conceito de número com a mensuração passou a ser menos intensa até desaparecer completamente. A escola pitagórica é conhecida por ter tido a concepção de que o número é a essência de todas as coisas. É razoável dizer que uma das razões de tal concepção ter surgido foi a observação pelos membros dessa escola da ocorrência de certos padrões na produção dos sons, isto é, em um instrumento musical de corda, quando a corda é diminuída ao meio, se obtém uma nota uma oitava acima. Assim, a razão de uma nota para sua oitava é 1:2. Do mesmo modo descobriram que a razão de uma nota para sua quinta e quarta é 2:3 e 3:4 respectivamente. Outra descoberta dos pitagóricos que certamente os instigou a formular a teoria de que o número é a essência de todas as coisas é a seguinte. Se se tenta construir uma uma figura plana a partir de linhas retas de medida 3,4 e 5, obtém-se a figura de um triângulo com um ângulo reto. Se, no lugar das medidas 3,4 e 5 forem colocadas outras medidas com a mesma razão dessas, obter-se-a uma figura similar. Certamente o conhecido teorema de Pitágoras foi consequência de tais observações. Assim, os pitagóricos devem ter começado a pensar que para obter conhecimento da natureza das coisas, como na harmonia e na geometria, lhes bastava estudar os números e a razões entre eles. Seguramente é esta a origem da acepção atual da palavra "razão".

O programa pitagórico, contudo, não tardou a encontrar uma dificuldade insuperável. Ao tentar descobrir a razão entre o lado do pentágono regular com sua diagonal os matemáticos pitagóricos não conseguiam encontrar nenhuma razão. Von Fritz argumenta que Hippasus de Metapontum ${ }^{1}$ foi o primeiro a demostrar a impossibilidade de encontrar uma razão. Ele demonstrou que não se consegue encontrar dois números que expressem a razão da diagonal com o lado do pentágono regular. Essa demonstração certamente causou um enorme abalo nas fundamentos da escola pitagórica, e não é de se estranhar que haja algumas lendas sobre a publicação de tal resultado por Hippasus. ${ }^{2}$ Posteriormente surgiram novos resultados de incomensurabilidade, em cujas provas apresentavam um grau de complexidade muito maior do que a de Hippasus, por exemplo, a demonstração que se o lado do quadrado fosse comensurável com a diagonal, um mesmo número seria par e ímpar.

O conhecimento da existência magnitudes para as quais não se conseguia encon-

\footnotetext{
${ }^{1}$ (Fritz, 1945, p. 260)

${ }^{2}$ Conta-se que Hippasus morreu em um naufrágio como punição pelos deuses por tornar públicos coisas tão terríveis. (ibid., p. 244)
} 
trar uma razão minou, portanto, a teoria das proporções dos pitagóricos, e junto com ela a tentativa de subsunção do conceito de magnitude ao conceito de número. A teoria das proporções dos pitagóricos somente se aplicava apenas às magnitudes comensuráveis. A busca de uma teoria das proporções que conseguisse englobar tanto as magnitudes comensuráveis como as incomensuráveis desembocou na teoria das proporções de Eudoxus. A teoria de Eudoxus claramente mostrava que na verdade a geometria é mais ampla que a teoria dos números, pois toda razão entre números pode ser expressa por uma razão entre magnitudes, mas não vice-versa.

Começaremos o texto fazendo uma exposição geral do conceito de número nos gregos desde os filósofos pré-socráticos, mostraremos em seguida como os pitagóricos tentam ampliar esse conceito de número, e como se viram impossibilitados, através da descoberta da incomensurabilidade, a levar a cabo tal tarefa. Posteriormente será apresentada a teoria das proporções que conseguia lidar com as magnitudes incomensuráveis, e como a diferença entre o conceito de número e magnitude é formulada por Aristóteles como uma diferença fundamental entre os conceitos de contínuo e discreto. Por fim, apresentaremos muito brevemente como o conceito de número foi ampliado posteriormente. ${ }^{3}$

\section{O conceito de número nos gregos}

O conceito de número nos gregos, que denominaremos aritmos, é completamente diferente do conceito de número atual, que engloba números naturais, números negativos, números racionais, números irracionais, números complexos, etc.

Para os gregos, o conceito de aritmos era ainda mais estrito que o conceito de número natural. Dois elementos do conjunto dos números naturais não estão presentes no conceito de aritmos: nem um, nem zero são aritmos. Que zero não fosse considerado um aritmos parece razoável, dada a concepção de aritmos como intimamente conectada com o processo de contagem, e não se conta zero coisas. Agora é possível contar uma coisa. Porque o um não era considerado um número entre os gregos? A explicação mais direta é que a unidade não era considerada aritmos porque ela era aquilo que constituía os aritmos, ou seja, aritmos é uma multiplicidade de unidades. Vejamos algumas definições de unidade:

- Iamblichus: de acordo "com alguns dos pitagóricos, uma unidade é o limite entre número e partes, porque dela [...] razões crescem reciprocamente em

\footnotetext{
${ }^{3}$ Está fora do escopo deste artigo o grande desenvolvimento da questão nos séculos 18-19, especialmente por Cauchy, Cantor, Dedekind, Peano e Frege.
} 
ambos os lados"; 4

- Theon: a unidade é "aquilo que, quando a multitude é diminuída por subtração contínua, é privado de todo número e toma uma posição duradoura e repousa" ${ }^{5}$

- Euclides define a unidade de uma forma bastante obscura: "Uma unidade é aquilo em virtude do qual cada coisa que existe é chamado um" ; 6

- Aristóteles define a unidade como um ponto sem posição. ${ }^{7}$

A definição de unidade mais clara é a de Aristóteles. Mas, embora seja um pouco melhor que as demais, percebe-se que a definição não ajuda muito. A definição de "ponto", dada nos Elementos ${ }^{8}$ é a seguinte: ponto é aquilo que não tem partes. Naturalmente, um ponto tem posição. Pela definição de Aristóteles, a unidade é aquilo que não tem partes nem posição. Supondo que essa definição delimita, ao menos vagamente, o significado de "unidade", o conceito de aritmos é facilmente definido:

- Nicomachus: "uma coleção de unidades"; "um fluxo de quantidade constituído de unidades"; 9

- Theon: "Um aritmos é uma coleção de unidades, ou uma progressão de multiplicidade começando a partir de uma unidade e uma retrogressão de multiplicidade cessando em uma unidade" $;{ }^{10}$

- Aristóteles: "uma multidão de indivisíveis";11 uma "Multidão mensurável por um". ${ }^{12}$

A concepção de aritmos como o resultado de uma medida faz com que a exclusão da unidade do conjunto dos aritmos seja bem razoável, visto que se a unidade é o

\footnotetext{
4 "some of the Pythagoreans, an unit is the boundary between numer and parts because from it, as from a seed and eternal root, ratios increase reciprocally on either side [...]". (Iamblichus, citado por (Euclid, 1956a, p. 279))

5 "That which, when the multitude is diminished by way of continued subtraction, is deprived of all number and takes an abiding position and rest". (Theon of Smyrna, citado por ibid.)

6 "An unit is that by virtue of which each of the things that exist is called one". (ibid., p. 277)

${ }^{7}$ (Barnes, 1984, Metaphysics, 1089b35)

${ }^{8}$ (Euclid, 1956b, p. 153)

9 "a collection of units"; "a flow of quantity made up of units". (Nicomachus, citado por ibid., p. 280)

10 "A number is a collection of units, or a progression of multitude beginning from an unit and a retrogression ceasing at an unit". (Theon, citado por ibid.)

${ }^{11}$ (Barnes, 1984, Metaphysics, 1053a30)

${ }^{12}$ (ibid., 1057a3)
} 
estalão, ela não pode ser o resultado da medida, por ela própria ser o padrão de medida. Aristóteles dá essa mesma explicação, vejamos:

"Um" evidentemente significa uma medida. E em todos os casos ele é alguma coisa subjacente com uma natureza distinta de sua própria, e.g. na escala um quarto de tom, em magnitude uma polegada ou pé, ou algo do tipo, em ritmo uma batida ou sílaba; e similarmente em peso ele é um peso definido; e da mesma forma em todos os casos, em qualidades uma qualidade, em quantidades uma quantidade (e a medida é indivisível, no caso anterior em tipo e no último aos sentidos); O que implica que um não é, em nenhuma instância, ele mesmo uma substância. E isto é razoável; porque o um significa a medida de alguma pluralidade, e número significa uma pluralidade que foi medida e uma pluralidade de medidas. Portanto, é natural que um não é um número; porque a medida não é medidas, mas ambos a medida e o um são pontos de partida. ${ }^{13}$

\section{O programa pitagórico}

\subsection{O número como essência das coisas}

Na chamada "escola pitagórica" o estudo da matemática deixou de ser um meio para fins práticos e se tornou um objeto de estudo por seu próprio valor. Muitas descobertas importantes em geometria, na teoria das proporções e harmonia tiveram lugar nessa escola. Não há registro que as teorias dos pitagóricos são provenientes de Pitágoras, visto que deste não restou nenhuma obra escrita. Algumas fontes antigas $^{14}$ atribuem as descobertas sobre harmonia não a Pitágoras mas aos chamados pitagóricos, como Hípasus, ao qual é atribuído o experimento utilizando discos de metal de mesmo diâmetro e diferentes espessuras para demostrar as relações de proporção entre a espessura do disco e a nota produzida por ele ao ser tocado.

Aristóteles relata que os pitagóricos foram os primeiros a fazer progredir os estudos relacionados com a matemática, e que descobriram que há uma relação muito

\footnotetext{
13 "One" evidently means a measure. And in every case it is some underlying thing with a distinct nature of its own, e.g. in the scale a quarter-tone, in magnitude a finger or a foot or something of the sort, in rhythms a beat or a syllable; and similarly in weight it is a definite weight; and in the same way in all cases, in qualities a quality, in quantities a quantity (and the measure is indivisible, in the former case in kind, and in the latter to the sense); which implies that the one is not, in any instance, in itself a substance. And this is reasonable; for the one means the measure of some plurality, and number means a measured plurality and a plurality of measures. Thus it is natural that one is not a number; for the measure is not measures, but both the measure and the one are starting-points.(Barnes, 1984, Metaphysics, 1087b33-1088a15)

${ }^{14}$ (Kirk, Raven, \& Schofield, 1994, p. 243-244)
} 
- Diego Pinheiro Fernandes -

próxima entre a harmonia e os números. Além disso, ele diz que os pitagóricos, tendo sido criados em um ambiente de tantas descobertas matemáticas, passaram a achar que os números eram o princípio de todas as coisas. Diz Aristóteles, dadas as descobertas relacionadas ao número e a harmonia, os pitagóricos “[...] supuseram que os elementos dos números eram os elementos de todas as coisas, e que todo o céu era um acorde e um número" ${ }^{15}$. Isso é confirmado por um fragmento do pitagórico Filolau: "E o certo é que todas as coisas que se conhecem têm número: pois sem ele nada se pode pensar ou conhecer". ${ }^{16}$

\subsection{A descoberta da incomensurabilidade}

A descoberta das relações numéricas existentes nas figuras geométricas era objeto de pesquisa intensa na escola pitagórica. O conhecimento de que uma figura composta por três linhas retas medindo cada uma 3, 4 e 5 unidades forma um triângulo com um ângulo reto certamente não é de origem grega, visto que é razoável pensar que tal conhecimento deveria ser um conhecimento básico de qualquer carpinteiro egípcio ou babilônio. Tal conhecimento lhe permitia, por exemplo, construir um esquadro. Mas na escola pitagórica esse conhecimento foi levado a uma generalidade ainda não existente, ${ }^{17}$ o conhecido teorema de Pitágoras permitia construir um triângulo retângulo a partir de qualquer medida de lado.

Não deve ter demorado muito tempo após a descoberta do teorema de Pitágoras para que os pitagóricos percebessem que não conseguiam encontrar uma razão exata entre a diagonal e o lado de um triângulo isósceles retângulo de lado 1. A partir de então os pitagóricos, como o Theon, começaram a investigar métodos que resultavam em sucessivas aproximações do valor de $\sqrt{2} .^{18}$

Uma forma de encontrar a razão entre duas magnitudes era o chamado método da subtração recíproca ou, como ficou conhecido posteriormente, o algoritmo de Euclides. A ideia do algoritmo é simples: tome duas magnitudes desiguais $A$ e $B$ quaisquer. Da maior $A$ subtraia a menor $B$ até que reste uma magnitude $C$ menor que $B$, agora, de $B$ subtraia $C$ até que reste uma magnitude menor que $C$. Deve se fazer esse procedimento até que se obtenha uma magnitude $X$ que subtraída de outra magnitude $Y$ não deixe resto. Essa magnitude $X$ pois é a medida comum entre $A$ e $B$, e a razão entre elas será a quantidade de múltiplos de $X$ contida em $A$ pela quantidade de múltiplos de $X$ contida em $B$.

\footnotetext{
15 (Barnes, 1984, Metaph. L. A 5 985b23)

16 (Kirk et al., 1994, p. 344)

${ }^{17}$ Essa asserção é questionada em (Neugebauer, 1969), pelo fato de haver evidências do conhecimento do teorema de Pitágoras nos babilônios, nos hindus e nos chineses.

${ }^{18}$ (Heath, 1981, p. 91)
} 
Contudo, de acordo com Von Fritz, ${ }^{19}$ Hípasus, um pitagórico, descobriu que era impossível encontrar a razão entre a diagonal de um pentágono regular e o lado, pois nunca se encontra uma medida comum entre eles, pelo algoritmo de Euclides. A partir de tal descoberta, o sentimento de que nem todas as razões entre magnitudes são exprimíveis em aritmos se tornou conhecimento, e isso deve ter provocado um abalo tão grande nas doutrinas das escolas pitagóricas que não é de se estranhar que surgiram tantas lendas com relação a publicação por Hípasus de sua descoberta. A ideia de Filolau de que todas as coisas que se conhecem tem número não mais poderia ser sustentada, daí a nomenclatura "irracional" dada às magnitudes incomensuráveis.

A teoria das proporções dos pitagóricos é basicamente aquela expressa no livro VII dos Elementos ${ }^{20}$ e a definição de proporção é:

VII, Def. 20 Números são proporcionais quando o primeiro é o mesmo múltiplo, ou a mesma parte, ou as mesmas partes, do segundo que o terceiro é do quarto. ${ }^{21}$

Essa teoria não consegue lidar com magnitudes incomensuráveis porque, dadas duas magnitudes incomensuráveis, uma nunca será o mesmo múltiplo de outra, visto não terem uma medida comum.

\section{Os incomensuráveis e a nova teoria das pro- porções}

A ideia dos pitagóricos de que todas as relações entre as coisas do mundo poderiam ser expressas como relações entre aritmos foi a pique com a descoberta da incomensurabilidade. Aristóteles quando se remete à escola pitagórica parece considerar tal ideia errada e insustentável:

A doutrina dos Pitagóricos apresenta, por um lado menos dificuldades que as anteriormente mencionadas, mas, por outro, contém outras que lhe são peculiares. De facto não conceber o número como capacidade para existir em separado remove muitas das consequências impossíveis: mas que os corpos sejam compostos de números, e que esse número deva ser matemático, é coisa impossível. Pois não é correto falar de mudanças

\footnotetext{
${ }^{19}$ (Fritz, 1945, p. 257)

${ }^{20}$ (Euclid, 1956a, p. 113)

${ }^{21}$ Numbers are proportional when the first is the same multiple, or the same part, or the same parts, of the second that the third is of the fourth. (ibid., p. 278)
} 
espaciais indivisíveis: e por muito que possa haver grandeza deste tipo, as unidades, pelo menos não tem grandeza; e como é que uma grandeza pode ser constituída por indivisíveis? ${ }^{22}$

A descoberta da incomensurabilidade fez com que ficasse bem estabelecida uma distinção que já era mais ou menos presente fora da escola pitagórica: que aritmos é algo distinto de magnitude. A descoberta por Eudoxus de uma nova teoria das proporções que pudesse lidar tanto com magnitudes comensuráveis (que eram correlacionáveis a aritmos), quanto com magnitudes incomensuráveis, consolidou o fato de que a teoria das magnitudes, a geometria era então mais ampla do que a teoria dos aritmos.

\subsection{A teoria das proporções de Eudoxus}

A teoria das proporções atribuída a Eudoxus é apresentada no livro $\mathrm{V}$ dos Elementos. ${ }^{23}$ A definição de razão e proporção do livro $\mathrm{V}$ é um tanto complicada. Tentaremos esclarecê-la.

A definição de razão é a seguinte:

Uma razão é um tipo de relação com respeito ao tamanho entre duas magnitudes do mesmo tipo. ${ }^{24}$

Depois Euclides define quando duas magnitudes estão na mesma razão:

Magnitudes são ditas estarem na mesma razão a primeira com a segunda e a terceira com a quarta, quando, se quaisquer equimúltiplos forem tomados da primeira e da terceira, e quaisquer equimúltiplos da segunda e quarta, os primeiros equimúltiplos igualmente excedem, ou são iguais ou estão aquém dos últimos equimúltiplos tomados na ordem correspondente. $^{25}$

E, finalmente, temos a definição de proporção entre magnitudes:

Sejam as magnitudes que têm a mesma razão denominadas proporcionais. ${ }^{26}$

\footnotetext{
${ }^{22}$ (Arist. Met. M 8, 1083b8, citado por ibid.)

23 (Euclid, 1956a, p. 112)

${ }^{24} \mathrm{~A}$ ratio is a sort of relation in respect of size between two magnitudes of the same kind. (ibid., p. 114)

${ }^{25}$ Magnitudes are said to be in the same ration the first to the second and the third to the fourth, when, if any equimultiples whatever be taken of the first and third, and any equimultiples whatever of the second and fourth, the former equimultiples alike exceed, are alike equal to, or alike fall short of, the latter equimultiples respectively taken in corresponding order. (ibid.)

${ }^{26}$ Let magnitudes which have the same ratio be called proportional. (ibid.)
} 
Essas definições são melhor entendidas com um exemplo. Representaremos os múltiplos de uma magnitude $A$ da seguinte maneira $A, 2 A, 3 A$, etc. Sejam as seguintes escalas de múltiplos de $A$ e $B$ :

\begin{tabular}{ccccc}
\hline $1 \mathrm{~A}$ & $2 \mathrm{~A}$ & $3 \mathrm{~A}$ & $4 \mathrm{~A}$ & $5 \mathrm{~A}$ \\
\hline 1 & 1 & 1 & 1 & 1 \\
$1 \mathrm{~B}$ & $2 \mathrm{~B}$ & $3 \mathrm{~B}$ & $4 \mathrm{~B}$ & $5 \mathrm{~B}$
\end{tabular}

E sejam as seguintes escalas de múltiplos de $P$ e $Q$ :

\begin{tabular}{ccccc}
1 & 1 & & 1 & + \\
$1 \mathrm{P}$ & $2 \mathrm{P}$ & $3 \mathrm{P}$ & $4 \mathrm{P}$ & $5 \mathrm{P}$ \\
\hline $1 \mathrm{Q}$ & $2 \mathrm{Q}$ & $3 \mathrm{Q}$ & $4 \mathrm{Q}$ & $5 \mathrm{Q}$
\end{tabular}

Podemos observar que $2 A$ excede $2 B$ assim como $2 P$ excede $2 Q$ e que para qualquer $n, n A$ excede $n B$ e $n P$ excede $n Q$. De acordo com a definição de proporção, podemos dizer que $A$ está para $B$ assim como $P$ está para $Q$, sejam $A, B, P, Q$ comensuráveis entre si ou não.

Agora vejamos se ocorre proporção entre as magnitudes $C, D, I, J$. Seja a escala de múltiplos de $C, D$ :

\begin{tabular}{|c|c|c|c|c|}
\hline $1 \mathrm{C}$ & $2 \mathrm{C}$ & $3 \mathrm{C}$ & $4 \mathrm{C}$ & 50 \\
\hline $1 \mathrm{D}$ & $2 \mathrm{D}$ & $3 \mathrm{D}$ & $4 \mathrm{D}$ & $5 \mathrm{D}$ \\
\hline
\end{tabular}

E seja a escala de múltiplos de $I, J$ :

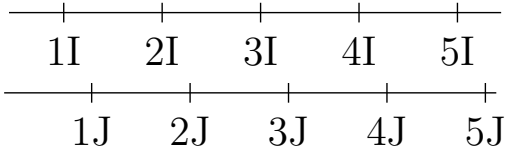

Veja-se que $2 C$ está entre $2 D$ e $3 D$, mas $2 I$ está entre $1 J$ e $2 J$, o que significa que $C$ não está para $D$ assim como $I$ está para $J$ : as magnitudes não estão em proporção.

Deve-se perceber o caráter infinitário dessa definição de proporção, visto que para saber que quatro magnitudes $A, B, P, Q$ estão em proporção, deve-se ser mostrado que todos os múltiplos de $A$ caem no mesmo intervalo dos múltiplos de $B$, que os múltiplos de $P$ nos intervalos dos múltiplos de $Q$. Podemos verificar que até o milionésimo múltiplo de $A$ a relação de proporção ocorre, mas só temos certeza absoluta até aí. Contudo, Heath explica ${ }^{27}$ que é possível superar essa dificuldade.

\footnotetext{
${ }^{27}$ (Euclid, 1956a, p. 124)
} 
- Diego Pinheiro Fernandes -

A ideia básica é que, ao investigar a relação de proporção entre quatro magnitudes $A, B, P, Q$, sejam tomados múltiplos genéricos dessas magnitudes e seja investigada a razão entre eles, fazendo um raciocínio por caso geral (o tipo de raciocínio é mesmo que ocorre quando se prova que determinada propriedade pertence a todos os triângulos, posto que pertence a um triângulo genérico).

\subsection{A existência de incomensuráveis como conhecimento básico}

A teoria das proporções de Eudoxus conseguiu resolver o problema que a descoberta das magnitudes incomensuráveis provocou na teoria das proporções anterior. A partir de então, algumas citações de Aristóteles e Platão nos dizem que o fato de que hajam magnitudes incomensuráveis era completamente "natural" e que deveria ser conhecido por todos. Platão chega a dizer que ele mesmo se envergonhava por apenas tarde na vida ter descoberto a ignorância deles sobre essas questões, ele diz:

[... Eu, como você, apenas tarde na vida soube com espanto da nossa ignorância nessas questões; para mim, nós parecemos ser mais semelhantes a porcos do que a homens, e eu estou bastante envergonhado, não só de mim mesmo, mas de todos os helenos. ${ }^{28}$

Pelo trecho não é possível dizer com certeza se Platão se envergonhava dele próprio desconhecer a existência de incomensuráveis até tarde na vida, ou se somente tarde na vida descobrir que os helenos em geral desconheciam essas coisas. Mas, de qualquer modo, fica claro que Platão considerava esse tipo de conhecimento como elementar, e que todos deveriam tê-lo. Mas adiante ele diz:

A natureza das quantidades comensuráveis e incomensuráveis nas relações de uma com a outra. Um homem que se preze deveria ser capaz, quando ele pensa, de distinguí-las; ${ }^{29}$

Também Aristóteles diz que o conhecimento da existência de magnitudes incomensuráveis só parece espantoso àquele que não compreendeu a prova de sua existência. Ele diz que seria na verdade muito espantoso que todas as magnitudes fossem comensuráveis. Diz Aristóteles:

\footnotetext{
${ }^{28}[\ldots]$ I, like yourself, have late in life heard with amezement of our ignorance in these matters; to me we appear to be more like pigs than men, and I am quite ashamed, not only of myself, but of all Helenes.(Plato, 1952, Laws, VII 819, p. 729)

${ }^{29}$ The nature of commensurable and incommensurable quantities in their relation to one another. A man who is good for anything ought to be able, when he thinks, to distinguish them; (ibid.)
} 
[...] pois parece maravilhoso para todos os homens que ainda não perceberam a explicação de que há algo que não pode ser medido nem pela menor unidade. [...] não há nada que surpreenderia um geômetra mais do que se a diagonal se mostrasse comensurável. ${ }^{30}$

\section{Aristóteles: quantidades contínuas e discretas}

Aristóteles, nas Categorias, faz uma distinção muito pertinente relacionada à questão dos incomensuráveis e a impossibilidade de representação aritmética. Ele argumenta que aritmos e magnitude pertencem a mesma categoria de quantidade, não obstante, são de espécie distinta: uma magnitude é algo contínuo e aritmos é algo discreto.

A distinção entre contínuo e discreto é feita da seguinte maneira. É discreto aquilo cujas partes não tem limite comum. Aristóteles dá o seguinte exemplo ${ }^{31}$ : cinco é parte de dez, mas dois cincos não se unem por nenhum limite comum para formar o dez, assim como o três não se une a um limite comum com o quatro para formar o sete. Outro exemplo de quantidade discreta que Aristóteles dá é da linguagem falada. Segundo ele, as partes da linguagem não se unem em nenhum limite comum: as silabas estão cada uma separadas em si mesmas.

As quantidades contínuas são várias: a linha, a superfície, o corpo, o tempo e o lugar. Nesse caso, cada um desses tem suas partes ligadas por um limite comum. Uma linha, por exemplo, quando dividida por um ponto, tem um limite comum entre as duas partes: o ponto divisor. A superfície, quando dividida pela linha, tem a linha como limite comum entre suas partes. O tempo também é assim, cada parte do tempo tem uma fronteira comum entre o tempo anterior e o tempo posterior.

Outra divisão feita por Aristóteles na categoria da quantidade é que em determinadas quantidades, como a linha, o plano, o sólido e o lugar, suas partes tem posição em relação ao todo, enquanto que em outras, como o número, o tempo e a linguagem, as partes não tem posição em relação ao todo. Um ponto tem certa posição em relação a outros pontos em uma linha, assim como uma linha tem posição em relação a outras linhas em um planto, e assim por diante. Com relação às partes de um número, percebe-se que isso não ocorre: três não está posicionado além ou aquém do dois. Com relação ao tempo, o argumento de Aristóteles é um pouco mais complexo. As duas premissas subentendidas no argumento são: para algo ter

\footnotetext{
${ }^{30}[. .$.$] for it seems wonderful to all men who have not yet perceived the explanation that there$ is a thing which cannot be measured even by the smallest unit.[...] there is nothing which would surprise a geometer so much as if the diagonal turned out to be commensurable.(Barnes, 1984, Metaphysics, A 983a13)

31 (Barnes, 1984, Categories VI, 4b25-30)
} 
- Diego Pinheiro Fernandes -

posição, ele deve perdurar; e nenhuma parte do tempo perdura. Disso se segue que nenhuma parte do tempo tem posição. Mas isso não significa que as partes do tempo não são ordenadas. Pode-se dizer que certa parte do tempo vem antes que outra. Um argumento semelhante é oferecido com relação ao caso da linguagem. ${ }^{32}$

Dadas essas considerações, efetivamente, a questão de se todas as quantidades contínuas podem ser expressas por quantidades discretas efetivamente parece descabida. Nesse sentido as citações de Aristóteles do fim da seção anterior parecem bem razoáveis.

\subsection{O contínuo não pode mesmo ser tornado discreto?}

O problema da relação entre contínuo e discreto está, naturalmente, na existência de quantidades incomensuráveis. As quantidades contínuas comensuráveis podem ser consideradas como discretas tomando-se uma unidade de medida comum, por exemplo duas linhas retas $A$ e $B$ podem ser tornadas discretas tomando um segmento $X$ que está contido em $A$ e $B m$ e $n$ vezes respectivamente, então se diz que $A=$ $m \times X$ e $B=n \times X$. Agora $m \times X$ e $n \times X$ são quantidades cujo resultado da operação de multiplicação resulta em um contínuo, mas que podem ser tratadas como discretas se se toma $X$ como o padrão de medida e se trabalha apenas com os números $m$ e $n$.

Contudo, no caso das quantidades incomensuráveis, não é possível encontrar uma quantidade que funcione como padrão de medida, e assim, não é possível torná-las discretas. De Morgan apresenta ${ }^{33}$ uma discussão interessante entre duas pessoas $A$ e $B$ sobre uma suposta solução, proveniente de uma ideia mais ou menos natural, acerca desse problema. A ideia é como se segue: como não é possível encontrar uma medida, por menor que seja, que não consiga medir o lado e a diagonal do quadrado? Afinal de contas, visto que é possível dividir a diagonal ad infinitum, deve haver alguma parte dessa divisão que seja capaz de medir o lado. "Não estaria então a linha toda absolutamente preenchida com pontos subdivisores, e não cortaria um deles uma linha igual ao lado do quadrado?" ${ }^{44}$, diz a personagem $A$. B então responde que a noção de incomensurabilidade foi definida por meio de uma noção finitista: tome a diagonal de um quadrado, divida qualquer número finito de vezes e o resultado nunca será capaz de medir o lado desse quadrado. Da possibilidade de dividir a diagonal ad infinitum naturalmente não se segue que a divisão já esteja feita atualmente. Se se consegue sustentar uma noção de infinito atual, com

\footnotetext{
${ }^{32}$ (ibid., 5a25-35)

33 (Morgan, 1836, p. 39)

34 "Would not the whole line then be absolutely filled with subdivision points, and would not one of them cut off a line equal to the side of the square" (ibid.)
} 
todos os problemas que ela acarreta, e considerar a digonal como completamente dividida em infinitas partes, então o problema da incomensurabilidade não desaparece, mas toma outra configuração. Haveriam certas magnitudes para as quais seria possível encontrar uma medida comum em finitos passos, e haveria outras que só se conseguiria achar uma medida comum em infinitos passos. Mas porque isso seria assim? Porque ainda existiria magnitudes comensuráveis e incomensuráveis: a distinção permaneceria.

Um contínuo é infinitamente divisível Além disso, Aristóteles já tinha argumentado no livro VI da Física quanto à impossibilidade de sustentar que algo contínuo seja composto por partes que, por sua vez, são indivisíveis, e.g. que a linha seja composta de pontos. O argumento dele é como se segue.

Os pontos que supostamente comporiam a linha somente poderiam estar relacionados entre si de três maneiras: (i) em continuidade um com o outro, (ii) em contato e (iii) em sucessão. Está em continuidade aquilo cujas partes tem um limite comum. Está em contato aquilo cujas extremidades estão juntas. Finalmente, estão em sucessão aquelas coisas em que não há nada de seu próprio tipo entre elas.

Suponha (i) que a linha seja composta de infinitos pontos em continuidade um com o outro. Mas dois pontos não podem ter a mesma extremidade, visto que o ponto é indivisível, ele não tem partes, e se não tem partes, ele não tem uma parte interna, e uma extremidade. Portanto, pontos não podem estar em continuidade um com o outro.

Suponha (ii) que a linha seja composta de infinitos pontos em contato. Se duas coisas estão em contato, ou estão em contato parte com parte, parte com todo, ou todo com todo. Visto que pontos não tem partes, eles só poderiam estar em contato todo com todo. Aristóteles diz de forma obscura que se os pontos estão em contato todo com todo, então eles não podem formar um contínuo, “[...] pois um contínuo é divisível em partes que são distinguíveis uma da outra no sentido de estarem em diferentes lugares" ${ }^{35}$. Bell ${ }^{36}$ nos ajuda a entender o que Aristóteles quer dizer, explicando que se uma quantidade de indivisíveis estão em contato completamente um com o outro eles colapsariam em um único indivisível, e não em um contínuo. Logo, pontos não podem estar em contato um com o outro.

Finalmente, suponha (iii) que a linha seja composta por infinitos pontos em sucessão. Mas dois pontos estão em sucessão quando não há nada de seu tipo entre eles. Mas entre dois pontos sempre há uma reta. E se por hipótese a reta é composta

\footnotetext{
35 "[...] for a continuum is divisible into parts which are distinguishable from each other in the sense of being in different places." (Barnes, 1984, Physics, VI, 1)

36 (Bell, 2005, p. 33)
} 
- Diego Pinheiro Fernandes -

de pontos, então há algo do mesmo tipo entre os dois pontos iniciais. Segue-se que dois pontos não podem estar em sucessão. Se for questionada a afirmação de que entre dois pontos sempre há uma reta, dizendo que nesse caso específico, os pontos estão tão juntos que não é possível descrever uma reta tendo eles como extremidade, então só é possível concluir que não haveria dois pontos, mas um só, e portanto, nenhum contínuo.

A suposição que a divisão de um contínuo deve chegar a um ponto final se mostra impossível. Portanto, um contínuo não pode ser formado por indivisíveis e, consequentemente, uma magnitude não pode ser tomada como composta de infinitos pontos.

\section{Considerações finais: de alguns desenvolvimen- tos posteriores do conceito de número}

Pouco a pouco a noção grega de aritmos foi sendo ampliada para a noção de número atual. Quanto a um algarismo para zero, sabe-se que provavelmente foi uma invenção hindú e há registros de sua utilização como marcador de lugar que datam do ano 876. ${ }^{37}$ Já quanto à utilização de um algarismo para zero no ocidente, há um registro em um museu inglês datando de 1300 em que o número zero é representado por $\phi .^{38}$

Na idade média já há argumentos para atribuição da qualidade de número à unidade. Um exemplo de argumento, atribuído a um matemático denominado Stevin (1585), de que a unidade tem que ser um número, é: se de um número $n$ for subtraído um não-número, o resultado é o próprio $n$; no entanto, $n-1$ é diferente de $n$, segue-se que 1 não é um não-número.

Quanto às frações, nem os gregos nem os romanos as consideravam como números. Nas atividades comerciais, quando era necessário a utilização de quantidades menores que dada unidade, eles tomavam submúltiplos da unidade e lhes davam nomes, por exemplo, ao invés de falar de meio quilo, se fala em 500g. ${ }^{39}$ Gradualmente a necessidade de um termo que expressasse de forma independente fez com que se passasse a considerar as frações unitárias como tendo um status de número. Frações como $\frac{2}{3}$, denominadas na idade média de "frações compostas" eram então representadas como a soma de duas frações unitárias $\frac{1}{2}+\frac{1}{6}$.

Após a inclusão das frações no conceito de número, as quantidades correlacionadas à magnitudes incomensuráveis, os irracionais, também passaram a ser

\footnotetext{
37 (Smith, 1958, p. 29)

38 (ibid., p. 78)

39 (ibid., p.208)
} 
considerados números. Newton, em 1769, já tinha a concepção de número atual, tomando indistintamente números naturais, números racionais e irracionais. Ele diz no começo de sua Aritmética Universal:

Por número nós entendemos não tanto uma multidão de unidades, mas sim a razão abstrata de qualquer quantidade, com outra quantidade do mesmo tipo, que nós tomamos por unidade. E isso pode ser de três modos: inteiro, fração e irracional: Um inteiro é aquilo que é medido pela unidade, uma fração é aquilo que um submúltiplo da unidade mede, e um irracional aquilo que é incomensurável com a unidade. ${ }^{40}$

Percebemos que, ao longo da história, o conceito de número foi ampliado por motivos práticos e teóricos. A utilização do zero como marcador de lugar naturalmente surgiu da necessidade de simplificar a representação numérica e as operações numéricas. Por outro lado, que a unidade seja considerada um número pode-se dizer que é por motivos teóricos de simplicidade da teoria dos números, etc. Com relação às frações, a sua consideração como entidades numéricas independentes parece ser atribuída tanto à necessidades práticas vinculadas à fatores econômicos, quanto à necessidade teórica de ser capaz de se falar em um número menor que a unidade, qualquer que seja a unidade, e.g., de ser capaz de usar o termo $\frac{1}{3}$ independentemente de qual é a unidade de medida. O desenvolvimento da álgebra na idade moderna não tardou a desencadear a junção dos conceitos de aritmos e magnitude (comensuráveis e incomensuráveis), formando o conceito que até então englobava todo tipo de número: o conceito de número real.

\section{Referências}

Barnes, J. (1984). The complete works of aristotle: the revised oxford translation. Princeton University Press.

Bell, J. (2005). The continuous and the infinitesimal in mathematics and philosophy. Polimetrica.

Euclid. (1956a). The elements (T. Heath, Ed.). Dover Publications. (volume 2) Euclid. (1956b). The elements (T. Heath, Ed.). Dover Publications. (volume 1) Fritz, K. V. (1945). The discovery of incommensurability by hippasus of metapontum. The Annals of Mathematics(2), 242-264.

\footnotetext{
${ }^{40}$ By number we understand not so much a multitude of unities, as the abstracted ratio of any quantity, to another quantity of the same kind, which we take for unity. And this is threeforld; integer, fracted, and surd: An integer, is what is measured by unity, a fraction, that which a submultiple part of unity measures, and a surd, to which unity is incommensurable.(Newton, 1769, p. 2)
} 
Do Conceito de Número e Magnitude na Matemática

Grega Antiga

- Diego Pinheiro Fernandes -

Heath, T. (1981). A history of greek mathematics: From thales to euclid (No. v. 1). Dover Publications.

Kirk, G. S., Raven, J. E., \& Schofield, M. (1994). Os filósofos pré-socráticos. Fundação Calouste Gulbenkian. (Trad. Fonseca, C. A. L.)

Morgan, A. (1836). The connexion of number and magnitude: an attempt to explain the fifth book of euclid. Taylor and Walton.

Neugebauer, O. (1969). The exact sciences in antiquity. Dover Publications.

Newton, I. (1769). Universal arithmetick: or, a treatise of arithmetical composition and resolution. Printed for W. Johnston. (Trad. Raphson, J. and Wilder, T.) Plato. (1952). The dialogues of plato. William Benton. (Trad. Benjamin Jowett)

Smith, D. (1958). History of mathematics. Dover Publications. (Vol. 2) 